\title{
Reliable and Robust Detection of Freezing of Gait Episodes With Wearable Electronic Devices
}

\author{
Ardian Kita, Paolo Lorenzi, Rosario Rao, and Fernanda Irrera
}

\begin{abstract}
A wearable wireless sensing system for assisting patients affected by Parkinson's disease is proposed. It uses integrated micro-electro-mechanical inertial sensors able to recognize the episodes of involuntary gait freezing. The system operates in real time and is designed for outdoor and indoor applications. Standard tests were performed on a noticeable number of patients and healthy persons and the algorithm demonstrated its reliability and robustness respect to individual specific gait and postural behaviors. The overall performances of the system are excellent with a specificity higher than $97 \%$.
\end{abstract}

Index Terms-Wearable electronic device, inertial sensors, freezing of gait, movement classification algorithms.

$\mathbf{T}$

\section{INTRODUCTION}$$
7
$$

HE implications of new technologies involving the use of sensors are becoming increasingly important in healthcare. This is the case of wearable sensors able to detect abnormal and/or unforeseen situations by monitoring physical and/or physiological parameters along with other symptoms [1]. The information that can be extrapolated from accelerometers and gyroscopes allows a correct reconstruction of the movements and a precise evaluation of the state of the musculoskeletal apparatus. The technological development and miniaturization of these devices has led to the possibility to be worn by patients who suffer from various diseases implying the motion sphere. The utility of their use in the patient care, assistance and rehabilitation consists in new and still not fully explored opportunities offered by the generation of big amounts of data regarding locomotion, postural and nocturnal disorders. Sensors can help monitoring and mitigating the effects of these disorders, customizing the therapy and eventually activating feedbacks to patients and care-givers.

Patients affected by the Parkinson's Disease (PD) can benefit mostly from the technological advancements in this field. PD manifests in about $1 \%$ of the worldwide population over 65 years, bringing severe ailments and disturbs related to the musculoskeletal apparatus, which include muscular rigidity, tremors, postural instability, bradykinesia, hypokinesia and akinesia [2]. These symptoms vary from one patient to another,

Manuscript received November 29, 2016; revised January 24, 2017; accepted January 24, 2017. The associate editor coordinating the review of this paper and approving it for publication was Dr. Wan-Young. Chung.

A. Kita, P. Lorenzi, and F. Irrera are with the Department of Information Engineering, Electronics and Telecommunications DIET, Sapienza University of Rome, 00184 Rome, Italy (e-mail: irrera@die.uniroma1.it).

R. Rao was with the Department of Information Engineering, Electronics and Telecommunications DIET, Sapienza University of Rome, 00184 Rome, Italy. He is now with Infineon, 35131 Padova PD, Italy.

Digital Object Identifier 10.1109/JSEN.2017.2659780

are very sensitive to the drug therapy and to the environmental inputs and depend on the progression of the disease. Today, the standard examination of the stage of the disease is done by doctors with the aid of patient and relative reports, which are generally incomplete and arbitrary.

In this context, it is easy to understand that a wearable electronic system for monitoring automatically and objectively the motion symptoms of PD patients is strongly desired. The processed data would help doctors in estimating better the stage of the disease and customize the therapy. The latter point is crucial to mitigate the symptoms. In fact, the proper therapy can reduce most of the symptoms, mainly at the early disease stage, and can help patients in preventing catastrophic falls as consequence of episodes of freezing of gait (FoG). FoG is defined as a paroxysmal block of movement associated with gait initiation, turning or negotiating an obstacle [3], [4], and can be accentuated by an incorrect drug therapy. FoG is described by the patients as a disabling symptom that makes their feet "stuck on the ground". In these situations, the patient reacts attempting to make the step, thus forcing the lower limbs and thrusting forward the trunk. For this reason, FoG is reported as the main cause of falls of PD patients [4], [5]. It has been demonstrated that a rhythmic auditory stimulation (RAS) as a metronome can release the involuntary block [6], [7]. Therefore, a wearable system able to provide a robust and reliable detection of the FoG in any context, and give timely a rhythmic auditory stimulation would be extremely useful.

As an evidence of the current interest in the field, several FoG detection systems have been proposed in literature in the last decade, to be used outdoor [8]-[10] or indoor [11]-[14]. They all employed inertial sensors disseminated on the patient body. Very recently, we too proposed a wearable wireless sensing system operating in real time [15]. Herein, that system will be called System 1. It used integrated micro-electromechanical (MEMS) inertial measurement units (IMU) to able to recognize specific kinetic features associated to motion disorders, typical of (but not limited to) the PD. The sensors were wireless connected to a PC. The algorithms provided detection and classification of the gait disorders using a time domain analysis of the data obtained through the fusion of the accelerometers and the gyroscopes signals. Then, the angular velocity and its low pass filter $\left(k_{\text {left }}, k_{\text {right }}\right)$ were calculated. The index $K$ given by the sum of $k_{\text {left }}$, and $k_{\text {right }}$ was finally compared with specific thresholds to classify regular states and disorders. System 1 was tested on 16 patients and performances in FoG detection were the best obtained to date. Notwithstanding, that system suffered by some severe 
limits which are now overcome by the system proposed here (System 2). First, the wireless communication between the sensors and the PC was lost whenever the maximum distance covered by the protocol communication was exceeded. To solve this problem, System 2 is designed to use a portable receiver (a smartphone), eventually connected with the home wireless LAN to transmit data to the PC. This also makes System 2 suitable for outdoor applications, with a battery life of a few hours. Second, the algorithm A1 exhibited problems in the FoG detection and classification in specific cases, as in the presence of noise sources related to the behavior of the $K$ index with time or to individual dubious gait and postural attitudes of patients. The algorithm A2 proposed here is robust respect to those sources of noise and its reliability is corroborated by a good statistic. The software platform is more generally suitable for the reconstruction of a visual skeletal representation of a moving human body.

\section{RELATED WORKS}

In literature, most of the reported work on the detection of FoG episodes is in the frequency domain. Mostly, the freezing index (FI) extrapolation has been used. It consists in evaluating the ratio between the power in the FoG band [2-6 Hz] associated to least leg tremor [16] and the power in the rest of the spectrum and comparing this ratio with defined thresholds. In this context, the first detection of FoG episodes was made monitoring the body acceleration with a 3-axis accelerometer [17]. They applied FFT, amplitude and wavelet analysis performing an offline processing. A few years later, Moore et al. [13] analyzed offline the accelerometer data collected on 11 patients. Authors detected the frequency components in the 3-8 Hz band during a FoG episode, which are not present in regular gait or voluntary rest. Calculating the FI, their algorithm obtained $89 \%$ accuracy and $89 \%$ sensitivity in FoG detection. Basing on the algorithm proposed in [13], other authors developed a system for online FoG detection [18]. That system contained three 3-axial accelerometers and a wearable computer. It was able to detect FoG episodes with user-dependent settings, exhibiting a sensitivity of $88.6 \%$, a specificity of $92.4 \%$ evaluated on a sample of ten patients, and a latency up to $2 \mathrm{~s}$. Manual adjustment of the algorithm parameters was necessary to achieve optimal results. Other online FoG-detection systems based on the FI extrapolation were presented in [19] and [20]. In the former work, authors used a 3-axis accelerometer and a wearable computer and detected FoG episodes with latency up to $580 \mathrm{~ms}$. In the latter work, authors studied a sample of 12 PD patients and evaluated the sensitivity in recognizing the occurrence of a FoG episode (reporting 100\% of success), without evaluating the sensitivity to timing and duration of each episode.

Remaining in the frequency domain, other methods of analysis alternative to the FI extrapolation have been developed. For example, the algorithm proposed in [12] based on the evaluation of the step length and cadence. Authors made a comparison with the FI extrapolation and concluded that their algorithm appeared more accurate in recognizing FoG episodes.
In pure time domain, the signal amplitude is considered rather than the frequency band, so that a low pass filter is needed to select the band of interest. This can be regarded as the main drawback of the time domain approach. On the other hand, this kind of analysis has the great advantage of performing a lower number of calculations, which reflects in a smaller power consumption and a longer battery life. Very few papers can be found in literature with the pure time domain approach. In this frame, we recall here the work by Y. Kwon et al. [21], which was based on the use of the root mean square (RMS) of the accelerometer signal, and our previous work [15], which was based on the fusion of raw accelerometers and gyroscope signals. Both detected FoG episodes through a threshold method. In [21], 20 patients were studied, obtaining a sensitivity and a specificity over $85 \%$. In [15], 16 patients were studied, obtaining a sensitivity and a specificity over $94 \%$.

Some work has been carried out in a combination of time and frequency domains, using different methods. Machine learning techniques were used by some authors [9], [22], [23]. Sensitivity and specificity higher than $98 \%$ have been reported in [22] on a sample of 10 patients, with a latency up to $710 \mathrm{~ms}$. In [24] fuzzy logic algorithms were applied reporting good sensitivity and specificity on 18 patients. Finally, very recently, S. Rezvanian et al. [25] proposed using the continuous wavelet transform (CWT) to define an index for identifying FoG episodes with good performances evaluated on 10 patients.

In conclusion of this Section, it is worth mentioning that all the work related to the detection of human body movements stems from the huge amount of work about the inertial navigation systems started in the second half of the XX century and still continuing today [26]-[28]. The most used signal fusion algorithm for the calculation of sensor orientation in navigation systems is the Kalman filter [29], while in our work we opted for the algorithm proposed by Mahony et al. [30], which is less computationally expensive and therefore more convenient for wearable applications. By comparing the two algorithms, we got negligible difference in the orientation estimation with a noticeable benefit from the calculation load viewpoint.

\section{The Starting Point: System 1, Algorithm A1}

In this Section, we will go through a summary of the features of System 1 and Algorithm A1 proposed in [15], which inspired System 2. System 1 consisted on a set of two IMU sensors, wireless connected to a PC collecting and processing data. The board used in System 1 is the same of System 2. It is a prototype called neMEMSi [31], [32], designed for processing signals in real-time and transmitting them. The IMU LSM9DS0 integrates a $\pm 16 \mathrm{~g}$ (g-force) $3 \mathrm{D}$ accelerometer, a \pm 12 Gauss $3 \mathrm{D}$ magnetometer and a \pm 2000 dps 3D gyroscope in a $4 \times 4 \mathrm{~mm} 2$ Land Grid Array package. A Bluetooth connection was used to transmit data. The BT33 class 1.5 micro-sized $(11.6 \times 13.5 \mathrm{~mm} 2)$ Bluetooth V3.0 module provided by Amp'ed RF/STMicroelectronics is a highly integrated solution for Bluetooth applications using the Serial Port Profile (SPP). The processing unit of neMEMSi 


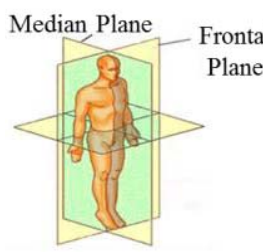

(a)

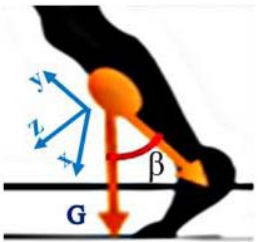

(b)

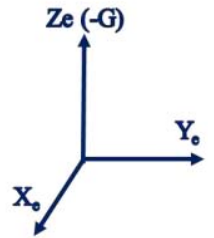

(c)

Fig. 1. Representation of the reference systems: (a) the median plane where the gait takes place; (b) the sensor reference system, with $G$ the gravity direction; and (c) the earth reference system, in which the sensor reference system rotates.

is the STM32L1, an ultralow- power 32-bit microcontroller provided by STMicroelectronics, with 33.3 DMIPS peak computation capability and an extremely low power consumption scalable down to $233 \mathrm{uA} / \mathrm{MHz}$. The Cortex ${ }^{\mathrm{TM} M} 3$ architecture along with the $32 \mathrm{MHz}$ clock frequency make this microcontroller suitable for advanced and low-power embedded computations. The board has a total dimension of $25 \times 30 \times 4 \mathrm{~mm}^{3}$ including the battery.

The detection and classification algorithm A1 used in System A1 was based on a time domain analysis of the sensors signals. The raw signals of accelerometers and gyroscopes are fused together by using an orientation estimation algorithm proposed by Mahony et al. [30]. To eliminate the gyroscope drift and to provide the sensor orientation in space, they used a correction vector provided by a Proportional Integral (PI) controller, where the error vector $\varepsilon$ driving the PI controller is determined from the previously estimated attitude and the accelerometer vector $a$. Authors suggested to use $\varepsilon=a \times d$ where $d$ is the direction of the gravity vector as given by the estimated attitude. Regarding the PI controller, the value of the integral coefficient is $K_{i}=0.0025$, while the proportional coefficient is $K_{p}=0.5$. A quaternion based representation of the limbs orientation and position was calculated and a 3D vector representing the limbs was generated. The sampling frequency $\left(f_{s}\right)$ was $60 \mathrm{~Hz}$.

The sensors were positioned on the shins. Gait direction was in the median plane represented in Fig.1a. The $x-y-z$ sensor reference system is sketched in Fig.1b. Fig.1c shows the XeYe-Ze earth reference system in which the sensor reference system rotates. Ze coincides with negative $\mathrm{G}$ axis. The angle $\beta$ sketched in Fig. $1 \mathrm{~b}$ is used for the FoG detection and it is calculated as the angle formed between two 3D vectors: the negative $y$-axis and the gravity axis $(G)$. It is worth noticing that the angle $\beta$ is solid and, therefore, does not lie in the median plane. To detect FoG and calculate all the gait statistics, we need to analyze the projection of the $\beta$ angle onto the median plane. In this way, any information on the rotation around the $\mathrm{G}$ axis is ignored. Eventual discontinuities of the $\beta$ angle when it changes the sign, and consequent problems in angle derivation, can be easily overcome by conventional mathematical techniques.

The angular velocities $\omega_{\text {right }}$, $\omega_{\text {left }}$ obtained after the $\beta$ angle derivation were used as the input for the FoG detection algorithm. That algorithm calculated the first order

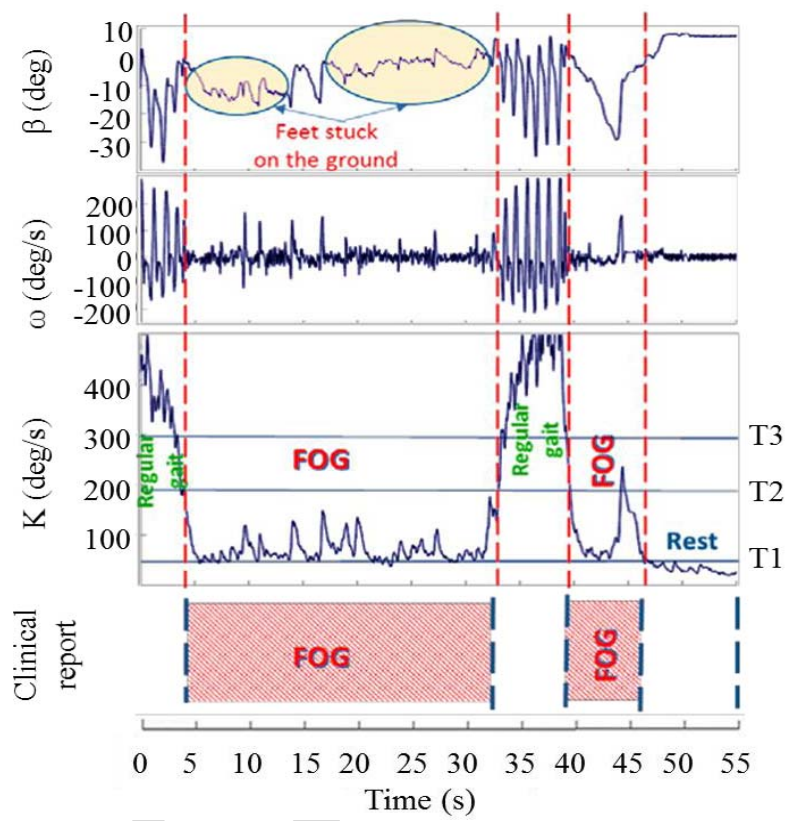

Fig. 2. Algorithm A1: representation of the angle (â), the angular velocity (ù) and $\mathrm{K}$ during a typical test. Our clinical absolute reference is also reported.

low-pass filtered angular velocities. We defined as $\omega_{t}$ and $k_{t}$, respectively, the right/left angular velocity and the lowpass filter measured at time $\mathrm{t}, k_{t-1}$ the value of $k$ at the previous step, $\alpha$ the smoothing coefficient set by the cutoff frequency $\left(f_{\text {cutoff }}\right)$ :

$$
\begin{aligned}
\text { kright } & =\text { lowpass }(\mid \text { wright } \mid) \\
\text { kleft } & =\text { lowpass }(\mid \omega l \text { lef } t \mid) \\
k t & =(1-\alpha) \cdot \omega t+\alpha \cdot k t-1 \\
\alpha & =(1+2 \pi \cdot \text { fcutoff } / f s)^{-1}
\end{aligned}
$$

In System 1, it was: $f_{\text {cutoff }}=0.83 \mathrm{~Hz}, f_{s}=60 \mathrm{~Hz}, \alpha=0.92$. Finally, the index $K$ was defined:

$$
K=k l e f t+k r i g h t .
$$

Patients were asked to wear the sensors and walk some steps, turn and go back. All the tests were filmed and the films were studied by doctors who determined the exact onset and ending times of the freezing episodes. Those clinical statements represented our absolute reference, which allowed to define three threshold values of the $K$ index (T1-T3) to classify four stationary states: regular gait $(K>\mathrm{T} 3)$, preFoG time (T3> $K>\mathrm{T} 2)$, FoG state $(\mathrm{T} 2>K>\mathrm{T} 1)$ and rest state $(K<\mathrm{T} 1)$. Once the values of T1-T3 were fixed for a certain patient, they remained unchanged for the whole duration of the monitoring.

Distinguishing correctly the involuntary block (i.e., the FoG) from the voluntary block is crucial because in real time a false negative (i.e., a FoG episode classified as a voluntary block) would not switch on the audio-feedback. At the same time, a false positive (i.e., a voluntary block classified as FoG) would switch on the audio feedback when not necessary, thus confusing the patient. In Fig. 2 we can see how the algorithm A1 works. In that test the patient was a female, 


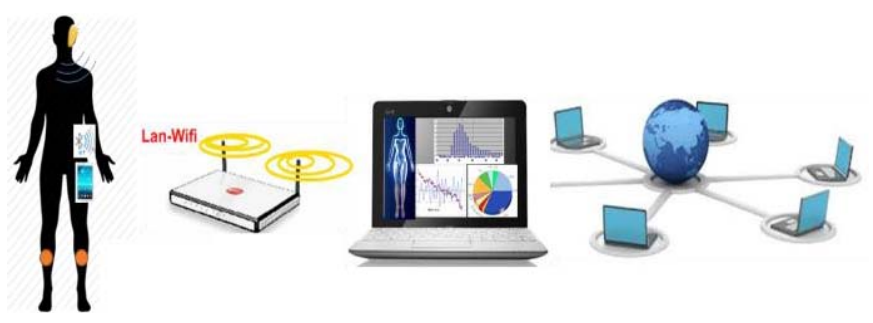

Fig. 3. Sketch of System 2: the two sensors are positioned on the shins, a smartphone is used as portable receiver, a headphone is wireless connected for the auditory feedback, a PC is connected to the smartphone via the wifi. The information and database can be shared in a cloud.

over 65 , in an advanced stage of the disease. The behavior of the angle $(\beta)$, the angular velocity $(\omega)$ and the $\mathrm{K}$ index are shown as function of the test time. As one can see, the $\beta$ and $\omega$ curves varied consistently in the different portions of the figure. In particular, it is easy to appreciate an oscillatory behavior of $\beta$ and $\omega$ during the regular gait $(0-4 \mathrm{~s} ; 32-39 \mathrm{~s})$ and a flatness during the rest state (46-55 s). The $K$ index exhibited a wide variability.

The clinical report by doctors about the exact FoG timing is indicated in the bottom. They referred the occurrence of two FoG episodes, between 4 and $32 \mathrm{~s}$ and between 39 and $46 \mathrm{~s}$. The comparison between the $\mathrm{K}$ index and the clinical report allowed defining the $\mathrm{T}$ thresholds for the state classification. A strength of this kind of systems is the possibility to distinguish between the rest state and the FoG thanks to the fact that during a FoG sensors are able to detect any least activity related to leg muscle contractions. To this regard, looking at Fig. 2 one can see that during the test the patient interrupted abruptly the regular gait for two times remaining involuntarily blocked with the feet stuck on the ground. During those time intervals, the sensors revealed the muscle contractions and the FoG episodes were correctly classified by the algorithm.

Algorithm A1 was tested on 16 patients, the time of each detected FoG was compared with the clinical reference. As a result, $94.5 \%$ sensitivity and $96.7 \%$ specificity were got [15].

\section{Upgrading The System: SyStem 2 , Algorithm A2}

System 1 suffered by a severe constraint imposed by the wireless communication between the sensors and the PC, when the maximum distance covered by the communication protocol was exceeded. System 2 releases that constraint thanks to the use of a portable receiver (a smartphone) and can be used outdoor for the real-time detection of FoG eventually giving an auditory stimulation. System 2 is sketched in Fig.3. It consists on the two sensors on the shins, a smartphone, a headphone for the auditory feedback and a PC for the data storage and processing. The information and database can be shared in a cloud. Using a smartphone, we set the sampling frequency $\left(f_{s}\right)$ to $25 \mathrm{~Hz}$. This has a benefit in that the number of transmitted data and the number of operations per unit time are lower than in the case at $60 \mathrm{~Hz}$, thus improving the sensors and smartphone battery life. In turn, setting $f_{s}=25 \mathrm{~Hz}$ does not present any drawbacks in the detection since the characteristic band of muscle tremors in PD lies well below $25 \mathrm{~Hz}$.

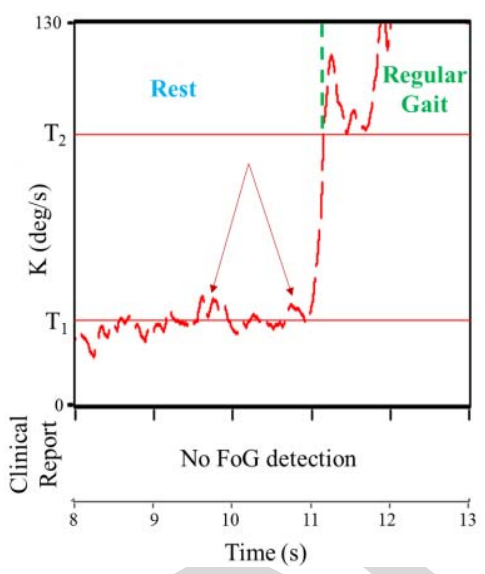

Fig. 4. Algorithm A1: Representation of typical fluctuations of the $\mathrm{K}$ index around T1 leading to "micro crossings" of the threshold.

From the soft viewpoint, a few problems had emerged with algorithm A1. Those issues and the solutions provided in algorithm A2 will be deeply discussed in the following. They regard: 1) unreliable identification of pre-FoG times; 2) micro-crossings of the thresholds, 3) slow variations of $\mathrm{K}$ during threshold crossings, 4) possible false FoG detection during body turning and 5) possible false FoG detection during body swing. For clarity, we will go through five intermediate steps, which will be called A2.1-A2.5, each addressing one of the issues listed before: the step A2.2 includes the solutions implemented in step A2.1, the step A2.3 includes the solutions implemented in step A2.2, and so on.

\section{A. Step A2.1 Against Unreliable Identification of Pre-FoG Times}

The first change is the elimination of the threshold T3 related to the identification of a pre-FoG time (T3> $K>\mathrm{T} 2$ ). The pre-FoG time was introduced in A1 to outline the transition between the regular gait and the FoG and vice versa, although it actually does not correspond to a state. The reason was that forecasting the FoG is highly desired for a timely feedback to the patient.

Unfortunately, the occurrence of pre-FoG episodes appeared extremely arbitrary, subject to a wide variability between one patient to another and also, for the same patient, between one test to another. Around 50\% of the tests revealed abrupt transitions between the two states while the other $50 \%$ revealed up to a few seconds in passing from one state to another. Furthermore, the risk that voluntary step shortening and slowdown were interpreted as pre-FoG was consistent. So, after a care evaluation of the whole set of tests, we concluded that the identification of a pre-FoG time was not reliable and also potentially dangerous for the patient. Therefore, in algorithm A2 the $K$ dynamics includes just two thresholds and three states: rest state, when $K$ lies in the interval [0-T1]; FoG state, when $K$ lies in the interval [T1-T2]; regular gait, $K>\mathrm{T} 2$.

\section{B. Step A2.2 Against False Classifications Due to Threshold Micro-Crossings of the K Index}

We define as "micro crossings" of the thresholds the fluctuations of the $K$ index around the values $\mathrm{T} 1$ and $\mathrm{T} 2$ which 


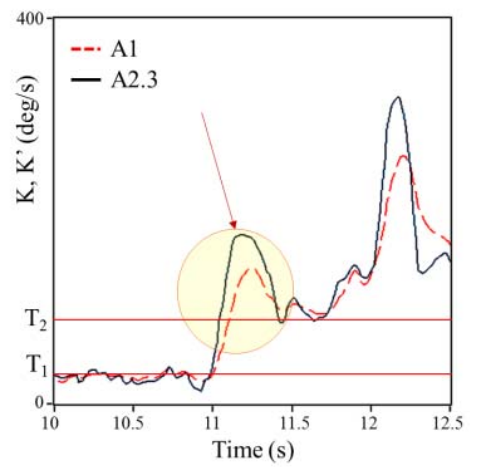

Fig. 5. Representation of the typical delay of the $K\left(K^{\prime}\right)$ index calculated with Algorithm A1 (A2.3) when crossing the threshold T1.

lead to classifications different from the real states of the patient. To elucidate the concept, we consider the $K$ index graph reported in Fig.4, obtained with algorithm A1. It relates to a patient who was first in the rest state and then started walking at time $11 \mathrm{~s}$. As one can see, during the rest state the $K$ index fluctuated around the $\mathrm{T} 1$ threshold (as outlined by the arrows). Algorithm A1 classified those time intervals as short FoG episodes, although the clinical reference did not. This is an example of false positive. We solve this problem in this step. Step A2.2 includes the activation of a waiting time ( $\left.t_{\text {wait }}\right)$ as soon as the $K$ index crosses one of the thresholds, at the end of which the value of $K$ is checked again. The state is classified after this procedure.

Two different waiting times are needed, depending whether getting out of the T1-T2 interval (FoG interval) or entering it. In fact, in the former case just one threshold will be crossed for sure (T1, if the patient releases the block going into the rest state, $\mathrm{T} 2$ if the patient releases the block starting walking), while in the latter case one or both thresholds will be crossed and the waiting time needs to be longer. The introduction of $t_{\text {wait }}$ implies a delay in the classification, which can be an issue if a FoG episode is occurring.

Therefore, the final choice of $t_{\text {wait }}$ should be a compromise between the necessity of a reliable classification and the maximum acceptable delay in FoG detection. In A2.2, we set $t_{\text {wait }}=100 \mathrm{~ms}$ when getting out the T1-T2 interval and $t_{\text {wait }}=$ $400 \mathrm{~ms}$ when getting into that range.

\section{Step A2.3 Against Slow Variations of K During Threshold Crossings}

We consider a zoom of Fig.4 in the time interval between $10 \mathrm{~s}$ and $12.5 \mathrm{~s}$. This is reported in Fig.5, with the red dashed curve (algorithm A1). As one can see, using A1 the transition of $K$ from T1 to T2 took a time around $100 \mathrm{~ms}$, which included the time constant $\tau=1 /\left(2 \pi \bullet f_{\text {cutoff }}\right)$ and corresponds to the time spanned by $K$ for a $3 \mathrm{~dB}$ variation. In algorithm A1 $f_{\text {cutoff }}$ was $0.83 \mathrm{~Hz}$ during the whole test time, regardless if the patient was in a stationary state or was making a transition between two states. To reduce this delay time, higher values of $f_{\text {cutoff }}$ would be desired. The new algorithm A2.3 introduces a mechanism that adapts the $\alpha$ coefficient in order to make $f_{\text {cutoff }}$ higher when $K$ crosses

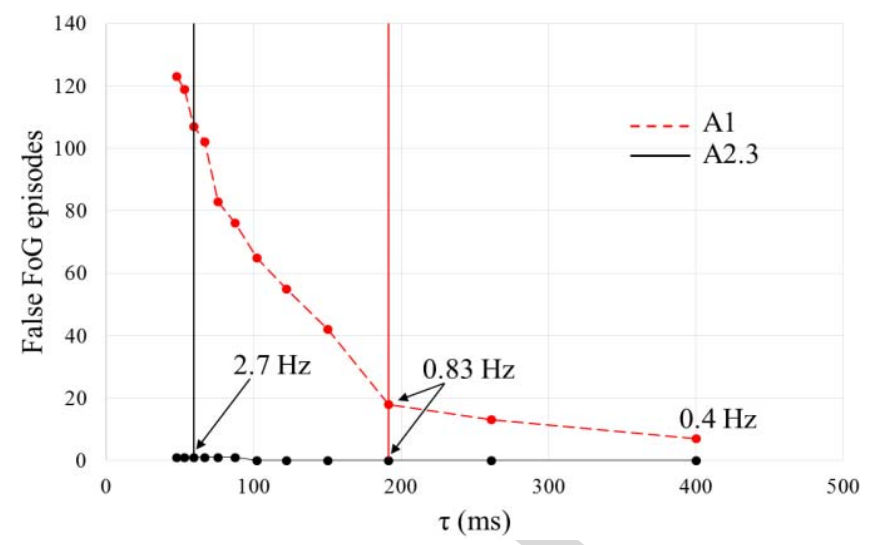

Fig. 6. The number of false FoG detections is plotted against the delay time of the FoG detection when the patient passed from regular gait to a FoG state, for the two algorithms. Points are calculated with different cutoff frequencies.

a threshold. However, increasing $f_{\text {cutoff }}$ the stability of the $K$ index degrades, meant as the fluctuations of $K$ around a threshold, which can induce false FoG detections. In Fig.6 the calculated number of false FoG detections in a real test is plotted against the time constant $\tau$, when the $K$ index entered the T1-T2 interval. The points correspond to different cutoff frequencies in the range $0.4-3.35 \mathrm{~Hz}$, with a $0.23 \mathrm{~Hz}$ step. Looking at the curve calculated with algorithm $\mathrm{A} 1$, one can see that raising $f_{\text {cutoff }}$, the high-frequency components of the $K$ index become more evident, increasing its instability and introducing many false FoG detections. On the other hand, $\tau$ is inversely proportional to $f_{\text {cutoff }}$, so a high value of $f_{\text {cutoff }}$ is desired to reduce delay.

As for the curve calculated with A2.3, its value is zero in most of that interval and starts raising for $f_{\text {cutoff }}$ above $\sim 2 \mathrm{~Hz}$. Thus the final choice of $f_{\text {cutoff }}$ in algorithm A2.3 is a trade-off between the need to have a short delay time in transitions and the need to have a stable $K$ index in the stationary states. In conclusion, we definitely set $f_{\text {cutoff }}=0.83 \mathrm{~Hz}$ (corresponding to $\alpha=0.827$ ) in stationary states and $f_{\text {cutoff }}=2.7 \mathrm{~Hz}$ (corresponding to $\alpha=0.6$ ) when passing thresholds. As a result, in Fig.5, the curve calculated with the algorithm A2.3 exhibits much shorter transition times than the other one.

It is worth noticing that the frequency $0.83 \mathrm{~Hz}$ falls below the characteristic interval of FoG frequencies [2-6Hz], and that the attenuation at $6 \mathrm{~Hz}$ is approximately $18 \mathrm{~dB}$. Although this attenuation seems rather high, it is necessary for the correct operation of the algorithm. In fact, we need to distinguish the FoG episode from both the rest state and the regular gait. As for the regular gait, its $\mathrm{K}$ amplitude is much higher than in FoG, as outlined in Fig.2, and the higher the attenuation in the FoG band the easier the capability of distinguishing the regular gait from the FoG. On the other hand, in the rest state we notice that in principle the $\mathrm{K}$ amplitude should be zero after low-pass filtering, apart from the eventual random drift of sensors. To this regard, we recall that the implementation of the fusion algorithm incorporated gyroscope bias drift compensation [28]. This implied that the random drift was always negligible apart from around the gravity axis. 
TABLE I

Values of the Adaptive Cutoff Frequency ( $\mathrm{FS}=25 \mathrm{~Hz}$ )

\begin{tabular}{ccc}
\hline \hline $\mathrm{K}$ & Action & $\mathrm{f}_{\text {cutoff }}(\mathrm{Hz})$ \\
\hline Stationary: $\mathrm{K}$ in $[0-\mathrm{T} 1]$ & Classify "rest state" & 0.83 \\
{$[0-\mathrm{T} 1] \rightarrow[\mathrm{T} 1-\mathrm{T} 2]$} & Wait 400ms \& verify & 2.7 \\
{$[\mathrm{~T} 1-\mathrm{T} 2] \rightarrow[0-\mathrm{T} 1]$} & Wait $100 \mathrm{~ms} \&$ verify & 2.7 \\
Stationary: $\mathrm{K}$ in $[\mathrm{T} 1-\mathrm{T} 2]$ & Classify "FoG" & 0.83 \\
{$[\mathrm{~T} 1-\mathrm{T} 2] \rightarrow[\mathrm{K}>\mathrm{T} 2]$} & Wait $100 \mathrm{~ms}$ \& verify & 2.7 \\
$\mathrm{~K}>\mathrm{T} 2 \rightarrow[\mathrm{T} 1-\mathrm{T} 2]$ & Wait 400ms \& verify & 2.7 \\
Stationary: $[\mathrm{K}>\mathrm{T} 2]$ & Classify “regular gait" & 2.7 \\
\hline \hline
\end{tabular}

However, also the latter drift contribution was filtered out by eliminating the component of limb rotation around the gravity axis, as it is not necessary for the algorithm. Furthermore, any residual drift coming from the accelerometer was filtered out too, by the fact that we based our calculations on the derivative of the angle. In conclusion, the $\mathrm{K}$ amplitude in the rest state is due only to sensor thermal and residual mechanical noise and lies typically $10 \mathrm{~dB}$ below the FoG K amplitude after low-pass filtering. So, distinguishing the FoG from the rest state in not a concern.

In Table I, the first column indicates the condition of $K$ (stationary or threshold crossing). In the second column, the algorithm actions are defined. The third column shows the corresponding values of $f_{\text {cutoff }}$ definitely used in A2.3 at $25 \mathrm{~Hz}$. In the bottom raw of Table I the stationary state with $K>\mathrm{T} 2$, classified as regular gait, is characterized by $f_{\text {cutoff }}=2.7 \mathrm{~Hz}$. This choice was made because there is the possibility that the patient suddenly stops voluntarily, causing an abrupt decrease of $K$, thus spanning on a wide dynamics. In this case, a lower cutoff frequency would reflect in a longer reaction time of the system. This is paid with a greater variability of $\mathrm{K}$ in the regular gait state, whose effects include some micro over-crossings of thresholds, which however are now ignored having introduced the waiting time in the step A2.2.

\section{Step A2.4 Against False FoG Detection}

\section{During Body Turning}

This problem may arise when the patient turns. In some case, body turning induces FoG, but more generally, body turning is accompanied by natural step shortening and movement slowdown. In any case, algorithm A1 classified those slow movements as FoG episodes, since $K$ remained in the interval T1-T2. To elucidate the concept, the red dashed curve in Fig.7a represents the $K$ index calculated with algorithm A1, during a patient turning (starting at time $\mathrm{t}=19 \mathrm{~s}$ ). Doctors reported that the patient experienced a FoG only at the end of the turning, whereas the algorithm A1 detected a FoG in the whole interval between the two red dashed lines.

To solve this problem, in the step A2.4 we introduce a turning coefficient, $K_{\text {turn }} . K_{\text {turn }}$ is calculated by considering the pure raw signal of the angular velocity around the sensor $\mathrm{y}$-axis only $\left(\omega_{\mathrm{Y}}\right)$, which corresponds to the negative G-axis
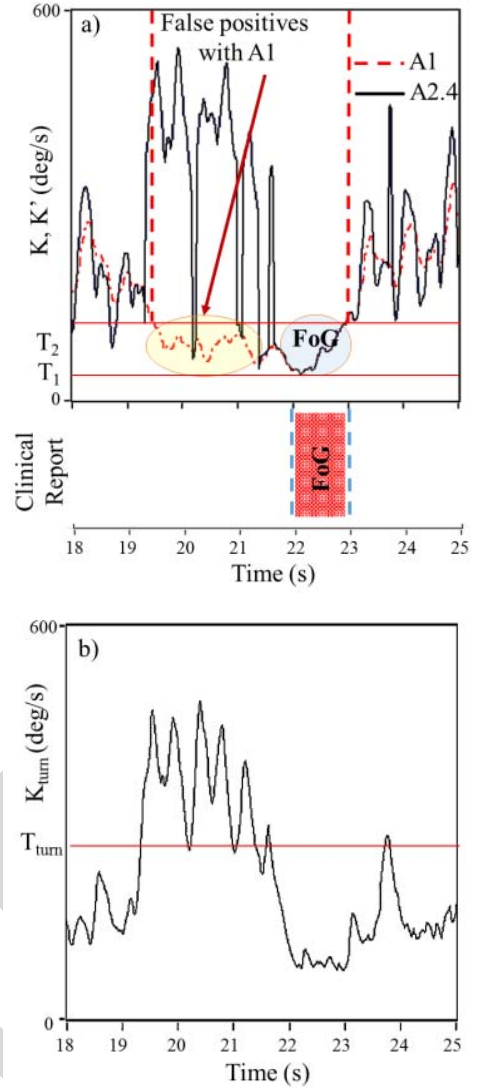

Fig. 7. (a) Curves of the $\mathrm{K}$ index obtained with algorithm $\mathrm{A} 1$ (dashed red line) and of the $\mathrm{K}$ ' index obtained with algorithm A2.4 (black continuous line), relative to a patient who turned after the time $t=19 \mathrm{~s}$. The clinical absolute reference is also reported. (b) Curve of the $\mathrm{K}_{\text {turn }}$ index in the same interval.

when the shin is at the vertical position (refer to Fig.1b):

$$
\text { Kturn }=\text { lowpass }(|\omega y|)
$$

The introduction of $K_{\text {turn }}$ is necessary since $K$ does not contain any information about the rotation around the $y$-axis. On the other hand, the accelerometer does not give any information during a rotation, so that in $K_{\text {turn }}$ it is not necessary to compute the fusion between gyroscope and accelerometer. Then, another threshold $\mathrm{T}_{\text {turn }}$ is defined, relative to $K_{\text {turn }}$. The $K_{\text {turn }}$ curve is displayed in Fig.7b in the same timescale of $K$. As one can see, $K_{\text {turn }}$ is always under the threshold $\mathrm{T}_{\text {turn }}$ apart from during the turning.

So, in algorithm A2.4 we define a new index:

$$
\begin{aligned}
& K^{\prime}=K+\text { KturnforKturn }>\text { Tturn } \\
& K^{\prime}=K \text { forKturn } \leq \text { Tturn }
\end{aligned}
$$

The curve of the $K^{\prime}$ index calculated with algorithm A2.4 is drawn in Fig.7a with the black continuous line. It correctly reports a short FoG only in the interval 22s $-23 \mathrm{~s}$.

\section{E. The New Algorithm: Step A2.5 Against False FoG Detection During Body Swing}

Here we define as body swing the oscillations of the trunk occurring in the frontal plane (Fig. 8). Body swing is a 


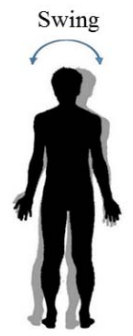

(a)

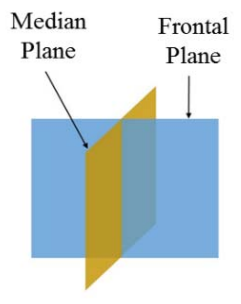

(b)

Fig. 8. Representation of the body swing in the frontal plane.

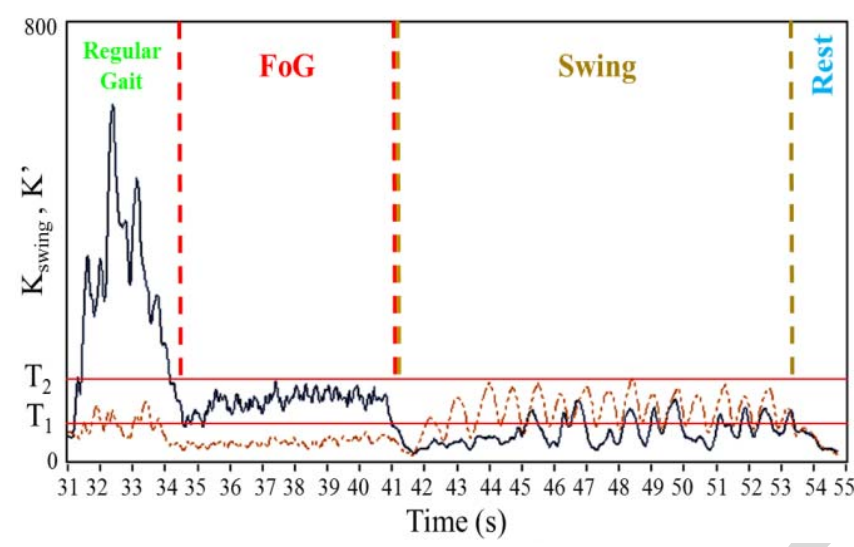

Fig. 9. Curve of the $K^{\prime}$ index (black continuous line) obtained with algorithm $\mathrm{A} 2$ and curve of the $\mathrm{K}_{\mathrm{swing}}$ index (brown dashed line) during a specific test performed on a healthy person oscillating the trunk.

recurrent postural habit of some people when they are in rest state, which is not related with any symptom of the PD.

During those oscillations a muscle activity is present in the inferior limbs, since the body weight switches from right to left. There is the risk that this muscle activity is erroneously interpreted as FoG. It is detected by the sensors on the shins as small variations of the gyroscope signal mainly on the z-axis.

In order to avoid that those rest states accompanied by least leg muscle activity were classified as FoG events, we define a new coefficient called $K_{\text {swing }}$ as the low pass filtered module of the raw gyroscope signal $\omega_{\mathrm{Z}}$ :

$$
\text { Kswing }=\text { lowpass }(|\omega z|)
$$

If $K_{\text {swing }}>K^{\prime}$, it is not a FoG episode. This procedure makes a comparison between the movements in the median and in the frontal plane sketched in Fig. 8. If the rotation in the frontal plane (around the sensor x-axis) is bigger than the rotation in the median plane (around the sensor z-axis), we are dealing with a body swing, not with a FoG.

We did not find any patient with the attitude of body swinging and the test was performed on healthy persons. The persons were asked to walk regularly, then to block and mimic a FoG, then to swing the body, then to rest.

In Fig.9 there are drawn the curves of $K^{\prime}$ (black continuous line) and $K_{\text {swing }}$ (brown dashed line) during a test. As one can see, in the body swing time (41s-53s) it is $K_{\text {swing }}>K^{\prime}$. In that time interval, the algorithm A2.5 does not report FoG,

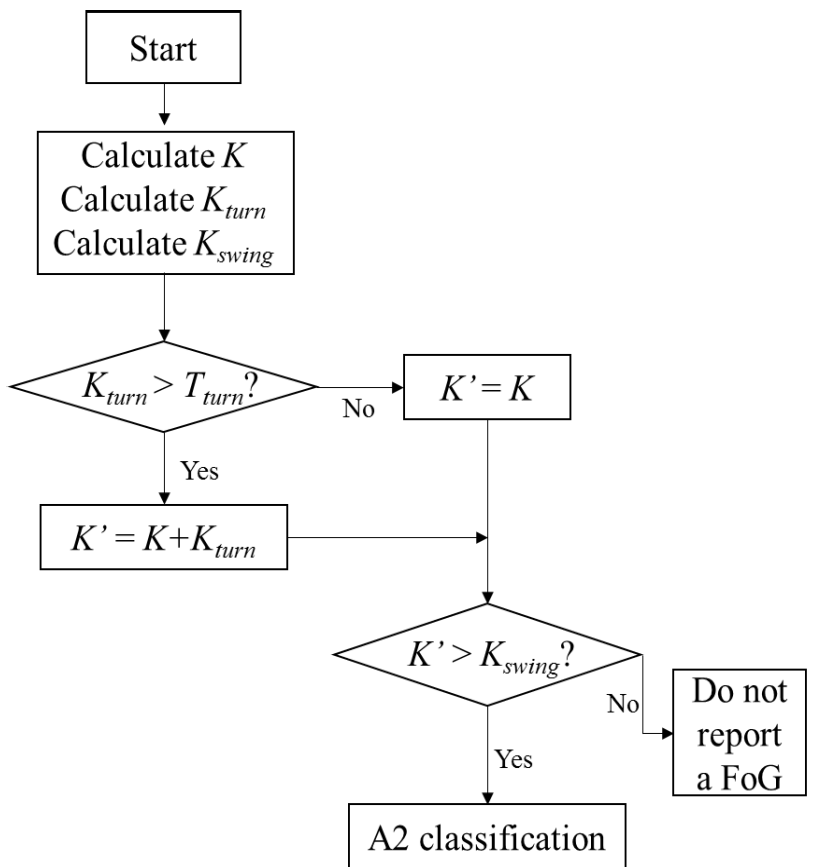

Fig. 10. Block scheme of Algorithm A2 operation.

TABLE II

Differencies Between Algorithms A1 AND A2

\begin{tabular}{|c|c|c|}
\hline Algorithm A1 & Algorithm A2 & Comment \\
\hline 3 thresholds & $\begin{array}{l}2 \text { thresholds } \\
\text { (step A2.1) }\end{array}$ & Abolished the pre- FoG state \\
\hline $\begin{array}{l}\text { Static threshold } \\
\text { evaluation }\end{array}$ & $\begin{array}{c}\text { Dynamic threshold } \\
\text { evaluation (step A2.2) }\end{array}$ & $\begin{array}{l}\text { Reduced false positives } \\
\text { in Ti micro- crossings }\end{array}$ \\
\hline Constant $f_{\text {cutoff }}$ & $\begin{array}{l}\text { Adaptive } f_{\text {cutoff }} \\
\text { (step A2.3) }\end{array}$ & $\begin{array}{l}\text { Reduced delay in } \\
\text { classification, false reports }\end{array}$ \\
\hline$K$ & $\begin{array}{l}K^{\prime}=K+K_{\text {turn }} \\
\text { (step A2.4) }\end{array}$ & $\begin{array}{l}\text { Reduced false reports in } \\
\text { body turning }\end{array}$ \\
\hline$K$ & $\begin{array}{l}K^{\prime} v s K_{\text {swing }} \\
\text { (step A2.5) }\end{array}$ & $\begin{array}{l}\text { Reduced false reports in body } \\
\text { swing }\end{array}$ \\
\hline
\end{tabular}

whatever the value of the $K^{\prime}$ index (the black curve). In the other intervals, it is always $K_{\text {swing }}<K^{\prime}$.

\section{F. Summarizing the Algorithm A2 Operation}

Algorithm A2 includes all the improvements discussed in the steps from A2.1 to A2.5. A block scheme of A2 operation is sketched in Fig.10. The algorithm initiates with the calculation of $K, K_{\text {turn }}$ and $K_{\text {swing }}$, as discussed in the previous sub-sections. Then $K_{\text {turn }}$ is compared with the threshold $\mathrm{T}_{\text {turn }}$ and only in the case $K_{\text {turn }}>\mathrm{T}_{\text {turn }}$ a new index $K^{\prime}$ is defined following eqs. $2 \mathrm{~b}$ and $2 \mathrm{c}$. Then, the new index $K^{\prime}$ is compared with $K_{\text {swing. }}$. If $K^{\prime}>K_{\text {swing }}$, then the algorithm A2 carries on the classification of the state, which does not include the possibility of a body swing. If not, the leg movement is interpreted as a body swing.

We conclude this Section with an overview of the differences between the two algorithms, listed in Table II. The five 
TABLE III

System Performances With Algorithms A1 AND A2

\begin{tabular}{ccccc}
\hline \hline $\begin{array}{c}\text { Average on 32 } \\
\text { patients }\end{array}$ & Specificity & Sensitivity & Precision & Accuracy \\
\hline A2 & $97.57 \%$ & $93.41 \%$ & $89.55 \%$ & $97.56 \%$ \\
A1 & $96.97 \%$ & $92.31 \%$ & $87.55 \%$ & $97.10 \%$ \\
Improvement & $+0.60 \%$ & $+1.10 \%$ & $+2.01 \%$ & $+0.46 \%$ \\
\hline \hline
\end{tabular}

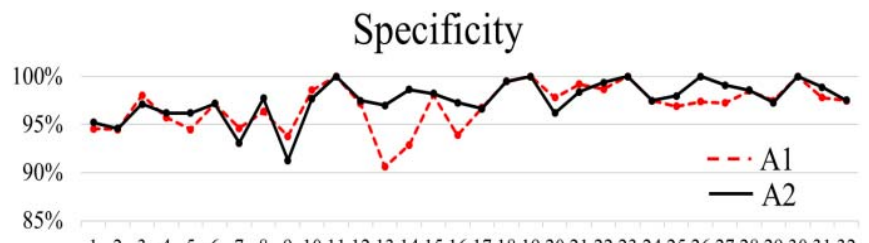

Patient \#

Fig. 11. The specificity of our system using algorithm A2 (black continuous line) or algorithm A1 (red dashed line) is drawn for each patient. Points are the average of four tests.

rows correspond respectively to the five changes operated in steps A2.1-A2.5, and the third column reports about each step achievement.

\section{RESULTS}

In this work, 32 patients have been studied ( 22 males and 10 females) whose age varied from 55 to 82 (average of 63). The test was the same for all of them and consisted on: an $8 \mathrm{mt}$ long walk, turning and walk back. Patients passed through an open door, which represented a virtual obstacle potentially inducing a FoG.

Each patient repeated the test several times, so that the total number of tests is 128 . We detected FoG episodes on 25 of the investigated patients. Table III resumes the average performance of the system in terms of specificity, sensitivity, precision and accuracy in FoG timing respect to the entire test time, for the two algorithms. In each test the clinical report was our absolute reference. The time of the FoG episodes detected by our system respect to the absolute reference was calculated. The two algorithms have been applied on the same dataset. As one can see, the average performances with algorithm A2 improved respect to A1.

In the case of patients exhibiting specific attitudes, the improvement obtained with algorithm $\mathrm{A} 2$ is much more consistent than the average value listed in Table III.

In fact, a few patients exhibited individual ways of walking and turning the body, which sometimes were mis-interpreted as FoG events by algorithm A1, but were correctly interpreted by algorithm A2. This is elucidated in Fig.11, where the specificity calculated with algorithm A2 is compared with that calculated with algorithm A1 for every patient. Each point of the plot corresponds to a single patient and is the average of four tests. Referring to Fig.11, algorithm A1 exhibited major problems with patients \#13, \#14, \#16, \#26. In details, patient \#13 had the habit to walk dragging the right leg, patient\#14 and \#26 slowed and shortened the steps while turning to almost stopping and, finally, patient \#16 stopped continuously while walking, probably because this helped him to concentrate on the steps. All those behaviors were sometimes mis-interpreted by algorithm A1, which in fact detected many more FoG events respect to the reality. On the contrary, those uncertain behaviors are now correctly interpreted by algorithm A2 thanks to the dynamic threshold evaluation, the adaptive cutoff frequency and the new parameter $K_{t u r n}$.

For all the other patients, algorithms A1 and A2 work similarly with very slight differences. Those minor differences are due to the fact that each patient exhibits FoG episodes of different duration: the same patient sometimes blocks for a fraction of second and some other times for many seconds. Now, when the FoG lasts around one second, the $400 \mathrm{~ms}$ delay introduced by algorithm A2 (A2.2) has a percentage effect which is not negligible, and worsen the FoG detection.

On the contrary, when the delay is much shorter than the block duration, the algorithm A2 works better than A1. In general, since the FoG time durations are not predictable a priori and are randomly distributed, the two curves in Fig.11 look very close with very slight positive or negative differences due to the statistical distribution of the FoG episode duration.

In conclusion, algorithm $\mathrm{A} 2$ is robust respect to possible noise sources introduced by individual patient attitudes. The only penalty in using algorithm A2 respect to A1 is the introduction of a delay of $400 \mathrm{~ms}$ in FoG detection. Of course, this is not a problem at all in off-line processing, since the resolution of our absolute reference is even longer. However, also in real time operation, in case that an auditory feed-back is to be given, a delay of $400 \mathrm{~ms}$ does not affect significantly the functioning.

Finally, to further verify the system reliability, we also performed 20 tests on 10 healthy persons. The healthy persons made the same exercise as the patients, voluntarily stopping sometimes during the walk, shortening and slowing down the steps, oscillating the body. As a result, no one FoG episode was classified with algorithm A2, obtaining the $100 \%$ specificity and accuracy in this set of tests.

\section{CONCLUSIONS}

A wearable wireless sensing system for assisting patients affected by Parkinson's Disease is proposed. It uses MEMS inertial sensors to recognize specific kinetic features associated to motion disorders as involuntary gait blocks, typical of (but not limited to) the PD. The system is designed for outdoor and indoor applications. Two sensors are positioned on the shins and are wireless connected to a portable receiver (a smartphone) which operates in real time and eventually provides an auditory stimulation to the patient in specific risky cases, as the involuntary Freezing of Gait episodes. The portable receiver can be connected with the home wireless LAN to transmit data to a PC, which operates offline for data storing and processing.

The proposed algorithm (A2) for the classification of the gait states is based on a time domain analysis. It makes a processing of the angular velocities calculated by operating a fusion between the accelerometer and the gyroscope signals. An index $K^{\prime}$ is obtained after low-pass filtering the angular velocities. The index $K^{\prime}$ is compared with thresholds defined 
after a preliminary calibration of the system, made through an absolute clinical reference.

Algorithm A2 starts from another algorithm (A1), respect to which it includes main changes devoted to the correct classification of the FoG episodes in the presence of noise. The noise sources treated in this work are due to minor behaviors in time of the $K^{\prime}$ index and to specific individual attitudes of some patients while walking, resting, turning. A dynamic evaluation of the thresholds reduces the false positive classifications of FoG in the case that the parameter $K^{\prime}$ shows micro-over crossings of the thresholds. A mechanism of adaptive cutoff frequency reduces the delay time in the classification of the gait states and reduces the occurrence of false positives and false negative classification of FoG episodes. A correction in the case of body turning reduces the possibility that steps shortening and movement slowdown are classified as FoG episodes. Finally, a correction in the case of body swing reduces the possibility that least leg movements due to body oscillations are classified as FoG episodes.

Repeated standard tests were performed on a group of $32 \mathrm{PD}$ patients of different age, gender and disease stage, and on a control group of 10 healthy persons. As a result, the overall system performances feature a specificity and a sensitivity of $97.6 \%$ and $93.4 \%$, respectively, were achieved on the patients group and a specificity and accuracy of $100 \%$ on the healthy control group. Algorithm A2 demonstrated robust with those patients exhibiting specific individual ambiguous attitudes while turning, walking or resting, where the previous algorithm A1 failed. Finally, we wish to notice that those performances are statistically meaningful thanks to the amount of persons monitored in this work.

\section{ACKNOWLEDGMENTS}

The authors are gratefully indebted to Dr. A. Suppa of the Department of Neurology and Psychiatry, Sapienza University of Roma, for precious advice and with STMicroelectrics (Agrate, Mi) for providing the electronic boards. Authors wish to thank also the patients who accepted to be involved in this work.

\section{REFERENCES}

[1] S. C. Mukhopadhyay, "Wearable sensors for human activity monitoring: A review," IEEE Sensors J., vol. 15, no. 3, pp. 1321-1330, Mar. 2015.

[2] J. Jankovic, "Parkinson's disease: Clinical features and diagnosis," J. Neurol., Neurosurgery Psychiatry, vol. 79, no. 4, pp. 368-376, 2008.

[3] A. Nieuwboer et al., "Abnormalities of the spatiotemporal characteristics of gait at the onset of freezing in Parkinson's disease," Movement Disorders, vol. 16, no. 6, pp. 1066-1075, Nov. 2001.

[4] M. Plotnik et al., "A motor learning-based intervention to ameliorate freezing of gait in subjects with Parkinson's disease," J. Neurol., vol. 261, no. 7, pp. 1329-1339, Jul. 2014.

[5] B. R. Bloem, J. M. Hausdorff, J. E. Visser, and N. Giladi, "Falls and freezing of gait in Parkinson's disease: A review of two interconnected, episodic phenomena," Movement Disorders, vol. 19, no. 8, pp. 871-884, Apr. 2004.

[6] P. Arias and J. Cudeiro, "Effect of rhythmic auditory stimulation on gait in Parkinsonian patients with and without freezing of gait," PLOS ONE, vol. 5, no. 3, p. e9675, 2010 .

[7] P. Arias and J. Cudeiro, "Effects of rhythmic sensory stimulation (auditory, visual) on gait in Parkinson's disease patients," Experim. Brain Res., vol. 186, no. 4, pp. 589-601, Apr. 2008.
[8] S. T. Moore, H. G. MacDougall, J.-M. Gracies, H. S. Cohen, and W. G. Ondo, "Long-term monitoring of gait in Parkinson's disease," Gait Posture, vol. 26, no. 2, pp. 200-207, Jul. 2007.

[9] S. Mazilu, M. Hardegger, G. Tröster, E. Gazit, and J. M. Hausdorff, "GaitAssist: A daily-life support and training system for Parkinson's disease patients with freezing of gait," in Proc. SIGCHI Conf. Human Factors Comput. Syst., Apr. /May 2014, pp. 2531-2540.

[10] M. Bächlin et al., "A wearable system to assist walking of parkinsons disease patients," Methods Inf. Med., vol. 49, no. 1, pp. 88-95, 2010.

[11] S. T. Moore et al., "Autonomous identification of freezing of gait in Parkinson's disease from lower-body segmental accelerometry,' J. Neuroeng. Rehabil., vol. 10, no. 1, pp. 19-29, 2013.

[12] B. Sijobert, J. Denys, C. A. Coste, and C. Geny, "IMU based detection of freezing of gait and festination in Parkinson's disease," in Proc. IEEE 19th Int. Funct. Elect. Stimulation Soc. Annu. Conf. (IFESS), Sep. 2014 pp. 1-3.

[13] S. T. Moore, H. G. MacDougall, and W. G. Ondo, "Ambulatory monitoring of freezing of gait in Parkinson's disease," J. Neurosci. Methods, vol. 167, no. 2, pp. 340-348, Jan. 2008

[14] G. Bonora, I. Carpinella, D. Cattaneo, L. Chiari, and M. Ferrarin, "A new instrumented method for the evaluation of gait initiation and step climbing based on inertial sensors: A pilot application in Parkinson's disease," J. Neuroeng. Rehabil., vol. 12, no. 1, pp. 45-57, 2015.

[15] P. Lorenzi, A. Kita, G. Romano, R. Rao, and F. Irrera, "Mobile devices for the real-time detection of specific human motion disorders," IEEE Sensors J., vol. 16, no. 23, pp. 8220-8227, Dec. 2016.

[16] J. M. Hausdorff, Y. Balash, and N. Giladi, "Time series analysis of leg movements during freezing of gait in Parkinson's disease: Akinesia, rhyme or reason?" Phys. A, Statist. Mech. Appl., vol. 321, nos. 3-4, pp. 565-570, Apr. 2003.

[17] J. H. Han, W. J. Lee, T. B. Ahn, B. S. Jeon, and K. S. Park, "Gait analysis for freezing detection in patients with movement disorder using three dimensional acceleration system," in Proc. 25th Annu. Int. Conf. IEEE Eng. Med. Biol. Soc., vol. 2, Sep. 2003, pp. 1863-1865.

[18] M. Bachlin et al., "Wearable assistant for Parkinson's disease patients with the freezing of gait symptom," IEEE Trans. Inf. Technol. Biomed., vol. 14, no. 2, pp. 436-446, Mar. 2010.

[19] E. Jovanov, E. Wang, L. Verhagen, M. Fredrickson, and R. Fratangelo, "deFOG-A real time system for detection and unfreezing of gait of Parkinson's patients," in Proc. Annu. Int. Conf. IEEE Eng. Med. Biol. Soc. (EMBC), Sep. 2009, pp. 5151-5154.

[20] M. D. Djuric-Jovicic, N. S. Jovicic, S. M. Radovanovic, I. D. Stankovic, M. B. Popovic, and V. S. Kostic, "Automatic identification and classification of freezing of gait episodes in Parkinson's disease patients," IEEE Trans. Neural Syst. Rehabil. Eng., vol. 22, no. 3, pp. 685-694, May 2014.

[21] Y. Kwon et al., "A practical method for the detection of freezing of gait in patients with Parkinson's disease," Clin. Interventions Aging, vol. 9, pp. 1709-1719, Oct. 2014.

[22] P. Bonato, D. M. Sherrill, D. G. Standaert, S. S. Salles, and M. Akay, "Data mining techniques to detect motor fluctuations in Parkinson's disease," in Proc. IEEE 26th Annu. Int. Conf. Eng. Med. Biol. Soc., Sep. 2004, pp. 4766-4769.

[23] S. Mazilu et al., "Online detection of freezing of gait with smartphones and machine learning techniques," presented at the in Proc. IEEE 6th Int. Conf. Pervasive Health, May 2012, pp. 123-130.

[24] L. Pepa, L. Ciabattoni, F. Verdini, M. Capecci, and M. G. Ceravolo, "Smartphone based Fuzzy Logic freezing of gait detection in Parkinson's Disease," in Proc. IEEE/ASME 10th Int. Conf. Mechatronic Embedded Syst. Appl. (MESA), Sep. 2014, pp. 1-6.

[25] S. Rezvanian and T. E. Lockhart, "Towards real-time detection of freezing of gait using wavelet transform on wireless accelerometer data,' Sensors, vol. 16, no. 4, p. 475, 2016.

[26] E. J. Lefferts, F. L. Markley, and M. D. Shuster, "Kalman filtering for spacecraft attitude estimation," J. Guid., Control, Dyn., vol. 5, no. 5, pp. 417-429, 1982.

[27] R. V. Garcia, N. F. O. Matos, H. K. Kuga, and M. C. Zanardi, "Unscented Kalman filter for spacecraft attitude estimation using modified Rodrigues parameters and real data," Comput. Appl. Math., vol. 35, no. 3, pp. 835-846, Oct. 2015.

[28] F. Zampella, M. Khider, P. Robertson, and A. Jiménez, "Unscented Kalman filter and magnetic angular rate update (MARU) for an improved pedestrian dead-reckoning," in Proc. IEEE/ION PLANS, Apr. 2012 , pp. 129-139.

[29] R. E. Kalman, "A new approach to linear filtering and prediction problems," Trans. ASME, D, J. Basic Eng., vol. 82, pp. 35-45, 1960. 

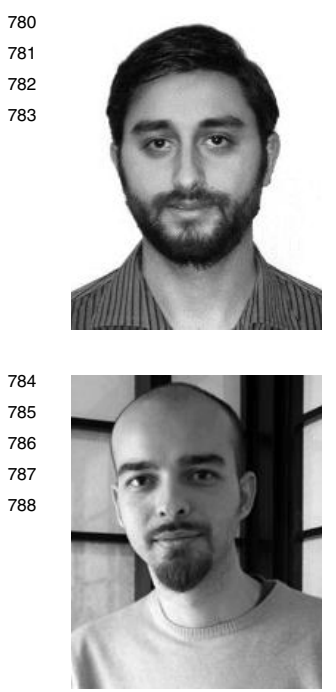

[30] R. Mahony, T. Hamel, and J.-M. Pflimlin, "Complementary filter design on the special orthogonal group SO (3)," in Proc. 44th IEEE CDC-ECC, vol. 5, Dec. 2005, pp. 1477-1484.

31] D. Comotti, M. Galizzi, and A. Vitali, "neMEMSi: One step forward in wireless attitude and heading reference systems," in Proc. Int. Symp. Inertial Sensors Syst. (ISISS), Feb. 2014, pp. 1-4.

[32] M. Caldara et al., "A novel body sensor network for Parkinson's disease patients rehabilitation assessment," in Proc. IEEE 11th Int. Conf. BSN, Jun. 2014, pp. 81-86.

Ardian Kita received the M.S. degree in nanotechnology engineering from Sapienza University of Rome in 2015. He is currently an assignee with the Sapienza University of Rome.

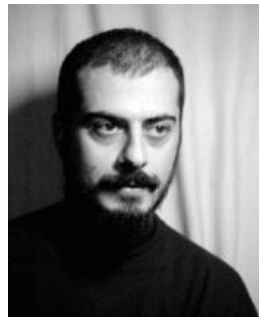

Rosario Rao received the Ph.D. degree in electronic engineering from the Sapienza University of Rome in 2011. From 2011 to 2014, he was an Assistant Professor with the Sapienza University of Rome. He is currently with Infineon, Padova.

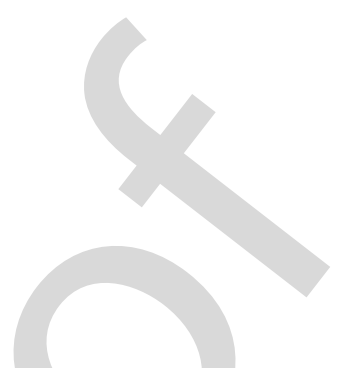

Paolo Lorenzi received the master's (cum laude) degree in electronic engineering from the Sapienza University of Rome in 2011, where he is currently pursuing the Ph.D. degree. From 2011 to 2012, he was with CEA-LETI, Grenoble.
Fernanda Irrera joined the Sapienza University of Roma in 1989, where she is currently a Professor of Integrated Electronic Components and responsible for the Micro- and Nano-Electronic Devices Laboratory. She also coordinates the IEEE-EDS Italy Chapter.

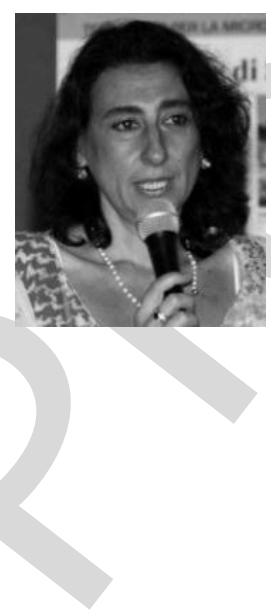




\section{AUTHOR QUERIES}

\section{AUTHOR PLEASE ANSWER ALL QUERIES}

PLEASE NOTE: We cannot accept new source files as corrections for your paper. If possible, please annotate the PDF proof we have sent you with your corrections and upload it via the Author Gateway. Alternatively, you may send us your corrections in list format. You may also upload revised graphics via the Author Gateway.

AQ:1 = Please confirm whether the edits made in the affiliation is set OK.

AQ:2 = Please note that references [4] and [13] are identical with [6] and [22], respectively. Hence we deleted refs. [6] and [22] and renumbered the other references. This change will also reflect in the citations present in the body text. Please confirm. 